\title{
Short- and long-term efficacy of aspirin and clopidogrel for thromboprophylaxis for mechanical heart valves: An in vivo study in swine
}

\author{
Stephen H. McKellar, MD, a,b Jess L. Thompson, III, MD, ${ }^{a}$ Raul F. Garcia-Rinaldi, MD, ${ }^{c}$ Ryan J. MacDonald, BS, ${ }^{a}$ \\ Thoralf M. Sundt, III, MD, and Hartzell V. Schaff, MDa
}

Objective: In the interest of exploring alternatives to warfarin, we tested the hypothesis that clopidogrel combined with aspirin is effective for thromboprophylaxis of mechanical valves using a swine model.

Methods: Adult swine underwent heterotopic implantation of a modified bileaflet mechanical valved conduit bypassing the ligated, native descending thoracic aorta. Animals were randomized to no anticoagulation $(\mathrm{n}=7), 175 \mathrm{U} / \mathrm{kg}$ dalteparin administered subcutaneously twice daily $(\mathrm{n}=9), 325 \mathrm{mg}$ of aspirin $(\mathrm{n}=6), 75 \mathrm{mg}$ of clopidogrel $(\mathrm{n}=6)$, or $325 \mathrm{mg}$ of aspirin and $75 \mathrm{mg}$ of clopidogrel daily $(\mathrm{n}=6)$ and survived for 30 days. Additionally, 11 animals were randomized to no anticoagulation $(\mathrm{n}=5)$ or $325 \mathrm{mg}$ of oral aspirin and $75 \mathrm{mg}$ of clopidogrel daily $(\mathrm{n}=6)$ and survived for 150 days.

Results: At 30 days, we observed $216 \pm 270 \mathrm{mg}$ of thrombus for the no anticoagulation group, $53 \pm 91 \mathrm{mg}$ for the dalteparin group, $33 \pm 23 \mathrm{mg}$ for the aspirin group, 25 $\pm 10 \mathrm{mg}$ for the clopidogrel group, and $17 \pm 9 \mathrm{mg}$ for the combined aspirin and clopidogrel group, respectively $(P<.01$ for clopidogrel and aspirin vs no anticoagulation). At 150 days, we observed $223 \pm 200 \mathrm{mg}$ of thrombus for the no anticoagulation group and $4 \pm 4 \mathrm{mg}$ for the aspirin and clopidogrel group $(P=.02)$. Mean platelet deposition on the valve was $4.1 \times 10^{9} \pm 3.6 \times 10^{9}$ for the no anticoagulation and $6.81 \times 10^{7} \pm 1.4 \times 10^{8}$ for the combined aspirin and clopidogrel groups, respectively $(P=.03)$. No major hemorrhagic events were observed.

From the Division of Cardiovascular Surgery, ${ }^{\mathrm{a}}$ Mayo Clinic, Rochester, Minn; the Clinician Investigator Program, ${ }^{\mathrm{b}}$ Mayo School of Graduate Medical Education, Mayo Clinic College of Medicine, Rochester, Minn; and the Advanced Cardiology Center, ${ }^{\mathrm{c}}$ Mayaguez, Puerto Rico.

Hartzell V. Schaff reports grant support from St. Jude; Thoralf M. Sundt III, reports consulting fees and grant support from St. Jude; Raul F. Garcia-Rinaldi reports lecture fees and grant support from St. Jude.

Received for publication Dec 3, 2007; accepted for publication Jan 28, 2008.

Address for reprints: Hartzell V. Schaff, MD, Division of Cardiovascular Surgery, Mayo Clinic, 200 First St SW, Rochester, MN 55905 (E-mail: schaff@mayo.edu).

J Thorac Cardiovasc Surg 2008;136:908-14 $0022-5223 / \$ 34.00$

Copyright $(2008$ by The American Association for Thoracic Surgery

doi:10.1016/j.jtcvs.2008.01.045
Conclusions: Effective short- and long-term thromboprophylaxis of mechanical valves can be achieved by using dual-antiplatelet therapy in this porcine model. Prospective human trials should be conducted with combination aspirin and clopidogrel as an alternative to warfarin in patients with bileaflet mechanical aortic valves.

$\mathrm{T}$ There is considerable interest in using new anticoagulation strategies for patients with mechanical heart valves because of the hemorrhagic and thromboembolic complications associated with long-term anticoagulation with warfarin. ${ }^{1}$ Several new classes of medications have been developed for long-term anticoagulation ${ }^{2,3}$ and have been studied clinically for conditions such as atrial fibrillation ${ }^{4}$ and the prevention and treatment of deep venous thrombosis after orthopedic surgery. ${ }^{5,6}$

Potent antiplatelet agents, such as clopidogrel, are among these newer agents. Clopidogrel is an orally administered adenosine diphosphate (ADP) P2Y12 receptor antagonist, which, in conjunction with aspirin, has demonstrated clinical benefit for a variety of cardiovascular conditions, including myocardial infarction ${ }^{7,8}$ and reduction of in-stent restenosis after coronary artery stenting. ${ }^{9,10}$

Dual therapy with aspirin and clopidogrel may have enhanced inhibition because each agent inhibits platelets through different pathways, the cyclooxygenase/thromboxane and ADP pathways, respectively. Clopidogrel combined with aspirin, however, has not been 


\section{Abbreviation and Acronym}

$\mathrm{ADP}=$ adenosine diphosphate

extensively studied in vivo for thromboprophylaxis of mechanical heart valves. We hypothesized that dual-antiplatelet therapy with aspirin and clopidogrel would be as effective as heparin for thromboprophylaxis of mechanical heart valves in swine.

\section{Materials and Methods}

\section{Animal Model and Study Design}

Approval for this study was obtained from the Animal Care and Use Committee of the Mayo Foundation. Adult swine underwent heterotopic mechanical aortic valve placement, as previously described. ${ }^{11}$ Briefly, the model consists of a modified bileaflet aortic valved conduit (St Jude Masters Series; St Jude, Minneapolis, Minn), which bypasses the ligated native descending thoracic aorta. This enables somatic blood flow distal to the left subclavian artery to pass across the mechanical valve in the newly constructed neoaortic (Figure 1). For the acute (30-day) study, we used the low-molecular-weight heparin dalteparin as the standard for anticoagulation because of the difficulty in maintaining a therapeutic window with warfarin in swine. ${ }^{12}$ For the chronic (150-day) study, the control group consisted of animals not receiving any anticoagulation.

\section{Acute Study}

Thirty-four animals were randomized into one of 5 treatment arms of postoperative anticoagulation. These groups consisted of no anticoagulation $(\mathrm{n}=7), 175 \mathrm{U} / \mathrm{kg}$ dalteparin (Eisai/Pfizer, Inc, Woodcliff, NJ) administered subcutaneously twice daily $(\mathrm{n}=9), 325 \mathrm{mg}$ of aspirin (Bayer, Inc, Pittsburg, Pa) administered by mouth daily $(\mathrm{n}=6), 75 \mathrm{mg}$ of clopidogrel (Bristol-Meyers Squibb/Sanofi Pharmaceuticals, New York, NY) administered by mouth daily $(n=6)$, or a combination of $325 \mathrm{mg}$ of aspirin and $75 \mathrm{mg}$ of clopidogrel administered by mouth daily $(n=6)$. Animals received their assigned treatment medication beginning on postoperative day 1 . Of note, data from 3 animals for both the no anticoagulation and dalteparin groups were pooled with data from previous experiments. ${ }^{13}$ The dose of dalteparin $(175 \mathrm{U} / \mathrm{kg}$ twice daily) was calculated from dosing studies performed in swine during a previous study. ${ }^{13}$

\section{Chronic Study}

Animals were randomly assigned to receive either no anticoagulation $(\mathrm{n}=5)$ or the combination of $325 \mathrm{mg}$ of aspirin and $75 \mathrm{mg}$ of clopidogrel administered by mouth daily $(n=6)$.

\section{Valve Thrombus}

The primary end point of this study was the amount of valve thrombus after 30 days for the acute study and after 150 days for the chronic study. Animals were sacrified after achievement of general anesthesia after systemic heparinization, at which time valve thrombus was measured and reported in milligrams as the mean \pm stan-

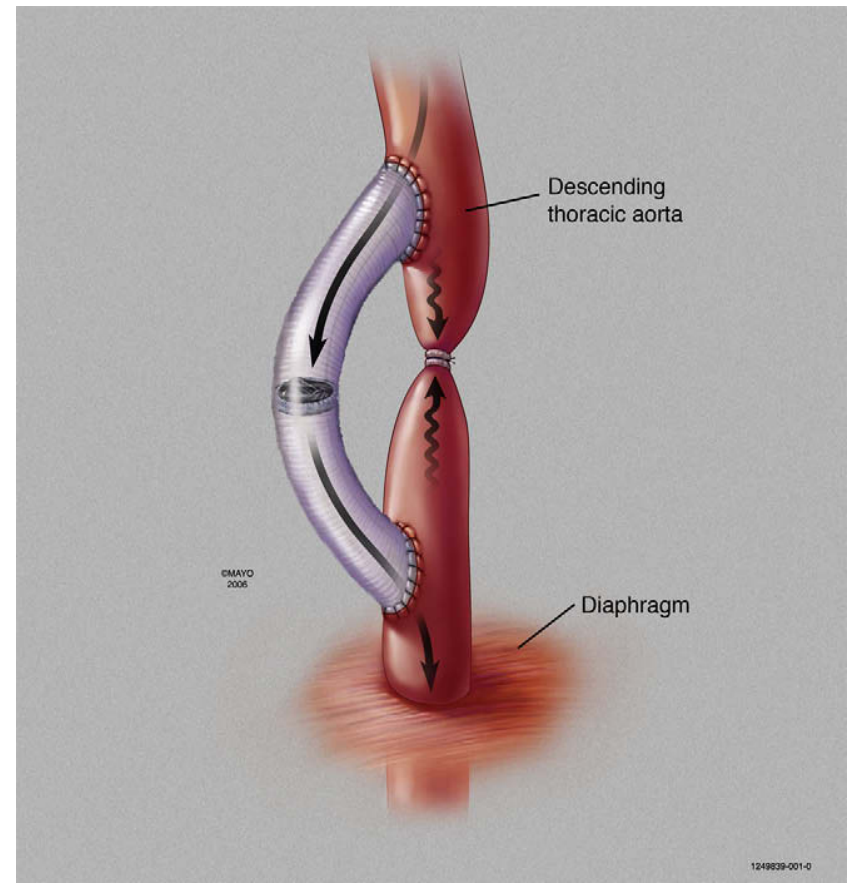

Figure 1. Heterotopic aortic valve model demonstrating the modified valved conduit (St Jude Masters Series, St Jude Medical) as it bypasses the ligated native descending thoracic aorta. Used with permission from the Mayo Foundation for Medical Education and Research.

dard deviation. Only thrombus on the valve was measured, and this was done blinded to the treatment arm.

\section{Hemorrhagic and Thromboembolic Complications}

Secondary end points were obvious or occult hemorrhagic or thromboembolic complications. Animals were observed daily for hemorrhagic complications (obvious gastrointestinal hemorrhage) and for thromboembolic complications (neurologic deficits or lower extremity or bowel ischemia). Additionally, kidneys were grossly inspected at the time of death to evaluate for gross embolic events. In the acute study occult hemorrhage was sought for as animals underwent serial blood draws to measure serum hemoglobin and fecal hemoglobin concentrations. Occult hemorrhagic events were defined as a decrease in the serum hemoglobin level of more than $2 \mathrm{~g} / \mathrm{dL}$ or an abnormally high fecal hemoglobin level ( $>2 \mathrm{mg}$ hemoglobin/g of stool).

\section{Platelet Aggregation Studies}

In the acute study platelet aggregation studies were performed by using platelet-rich plasma with a turbidimetric method, with a whole-blood aggregometer in optical mode (Chrono-log, Havertown, Pa). Arachidonic acid $(1.0 \mathrm{mmol} / \mathrm{L})$ and ADP $(10 \mu \mathrm{mol} / \mathrm{L})$ were used as agonists for aggregation, as described previously, ${ }^{14}$ to document platelet inhibition to aspirin and clopidogrel, respectively. 


\section{Platelet Deposition}

Platelet deposition on the mechanical prosthesis in the chronic study was assessed by using autologous platelet labeling with ${ }^{111}$ indium, as previously described. ${ }^{8,9}$ Briefly, after explantation of the valve, it was placed into a scintillation radiocounter (Capintec, Inc, Ramsay, $\mathrm{NJ}$ ). Background radioactivity and the amount of radioactivity from two 10-mL tubes of blood were obtained at the time of animal death. By using the animal's platelet count and radioactivity obtained from the tubes of blood, the average amount of radioactivity per platelet was determined. This was used to calculate the number of platelets on the prosthesis by using the following equation: Number of platelets on valve prosthesis $=$ Valve radioactivity/Platelet radioactivity.

\section{Statistical Analysis}

Valve thrombus and number of platelets deposited on the valve prosthesis reported in milligrams as the mean \pm standard deviation were treated as continuous variables. Mean valve thrombus and maximum platelet aggregation among the groups in the acute study were compared by using the Kruskal-Wallis test for heterogeneity. Mean valve thrombus for the antiplatelet groups was compared with that for the dalteparin group by using the Wilcoxon rank sum test.

\section{Results}

\section{Acute Study}

There were no perioperative deaths, and all animals survived for 30 days without obvious hemorrhagic or ischemic complications. One animal in the clopidogrel group had infectious valvular vegetation found at death (histologically confirmed, not shown) and was excluded from the analysis of mean thrombus weight. At the time of death, the mean thrombus weight for the groups was $216 \pm 270 \mathrm{mg}$ for the no anticoagulation, $53 \pm 91 \mathrm{mg}$ for the dalteparin, $33 \pm 23$ $\mathrm{mg}$ for the aspirin, $25 \pm 10 \mathrm{mg}$ for the clopidogrel, and 17 $\pm 9 \mathrm{mg}$ for the combined aspirin and clopidogrel groups, respectively (Figure 2,A). This represented a 10-fold decrease in mean valve thrombus for the combined aspirin and clopidogrel group than that seen in the no anticoagulation group $(P<.01)$. Although not statistically significant, we observed a trend in decreased thrombus formation in the combined aspirin and clopidogrel group compared with the other antiplatelet arms, suggesting a synergistic antiplatelet effect $(P=.5$; Figure 2, $B)$. Maximal platelet aggregation in platelet-rich plasma to ADP $(10 \mu \mathrm{mol} / \mathrm{L})$ decreased significantly with combination antiplatelet therapy with aspirin and clopidogrel $(P=.03)$, as shown in Figure 3, A. Representative postmortem photographs of explanted valves from the no anticoagulation and combined aspirin and clopidogrel groups are shown in Figure 4, $A$ and $B$.

There were no obvious hemorrhagic or thromboembolic complications. However, occult hemorrhagic complications were observed in 3 animals. One animal receiving dalteparin experienced an occult decrease in serum hemoglobin level from 12.4 to $6.5 \mathrm{~g} / \mathrm{dL}$. Two animals had abnormally high fecal hemoglobin assays of 3.5 and $15.6 \mathrm{mg}$ hemoglobin/g of stool, from the aspirin and dalteparin groups, respectively.
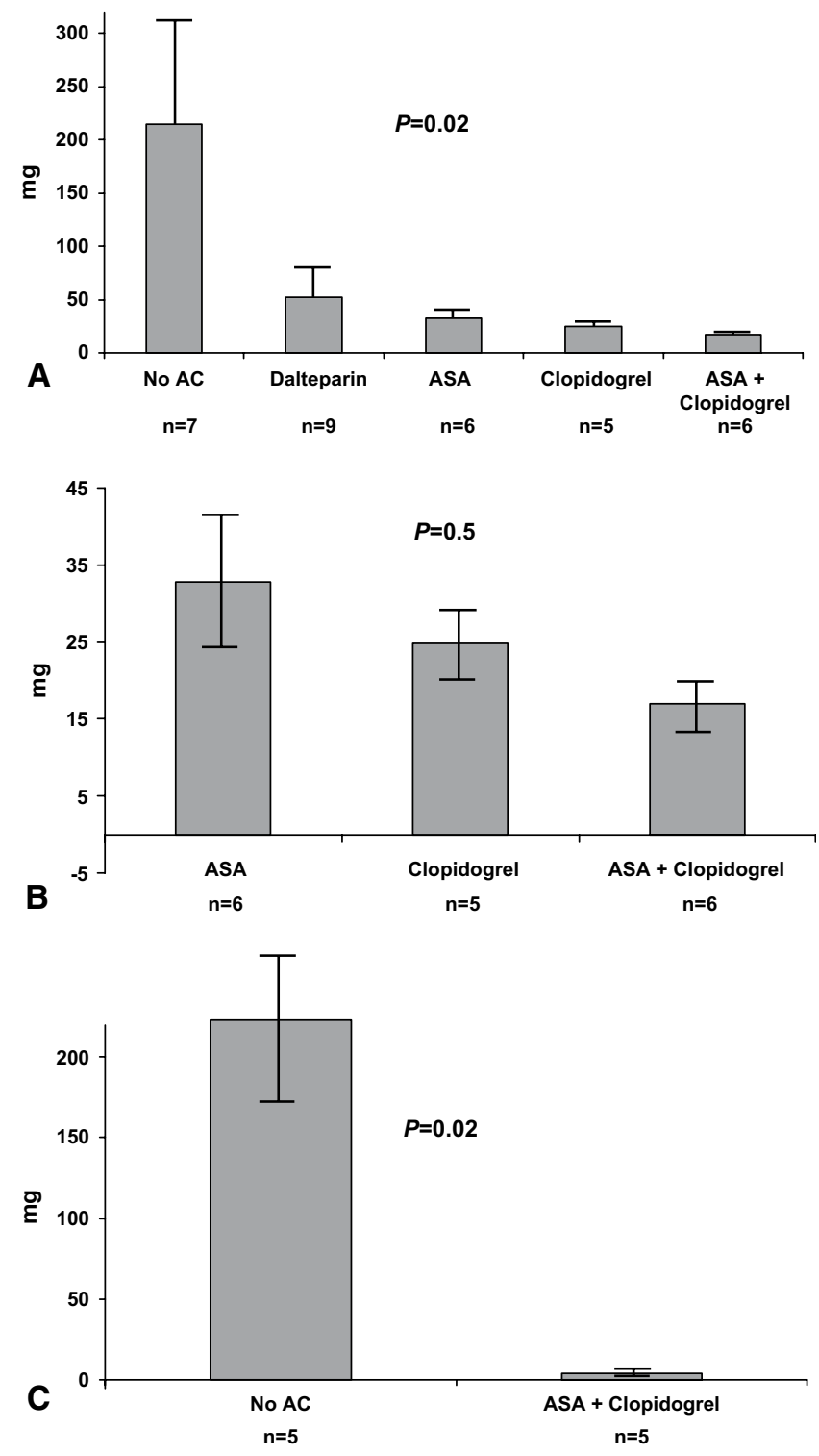

Figure 2. A, Mean valve thrombus (in milligrams \pm standard error of the mean) for each of the 5 arms at 30 days $(P=.02)$. $B$, Mean valve thrombus for the 3 antiplatelet arms at 30 days $(P=.5)$. Qualitatively, animals receiving combined aspirin and clopidogrel had the least amount of valve thrombus. C, Mean valve thrombus (in milligrams \pm standard error of the mean) for the two 150-day survivor groups: no anticoagulation and combined aspirin and clopidogrel $(P=.02)$. $n$, Number of animals; no $A C$, no anticoagulation; $A S A$, aspirin.

\section{Chronic Study}

There were no perioperative deaths, but 2 animals receiving aspirin and clopidogrel did not survive 150 days. One animal had signs of sepsis and was sacrified on postoperative day 85 . Infectious vegetations were seen on the valve (confirmed with histology, not shown). This animal was not included 

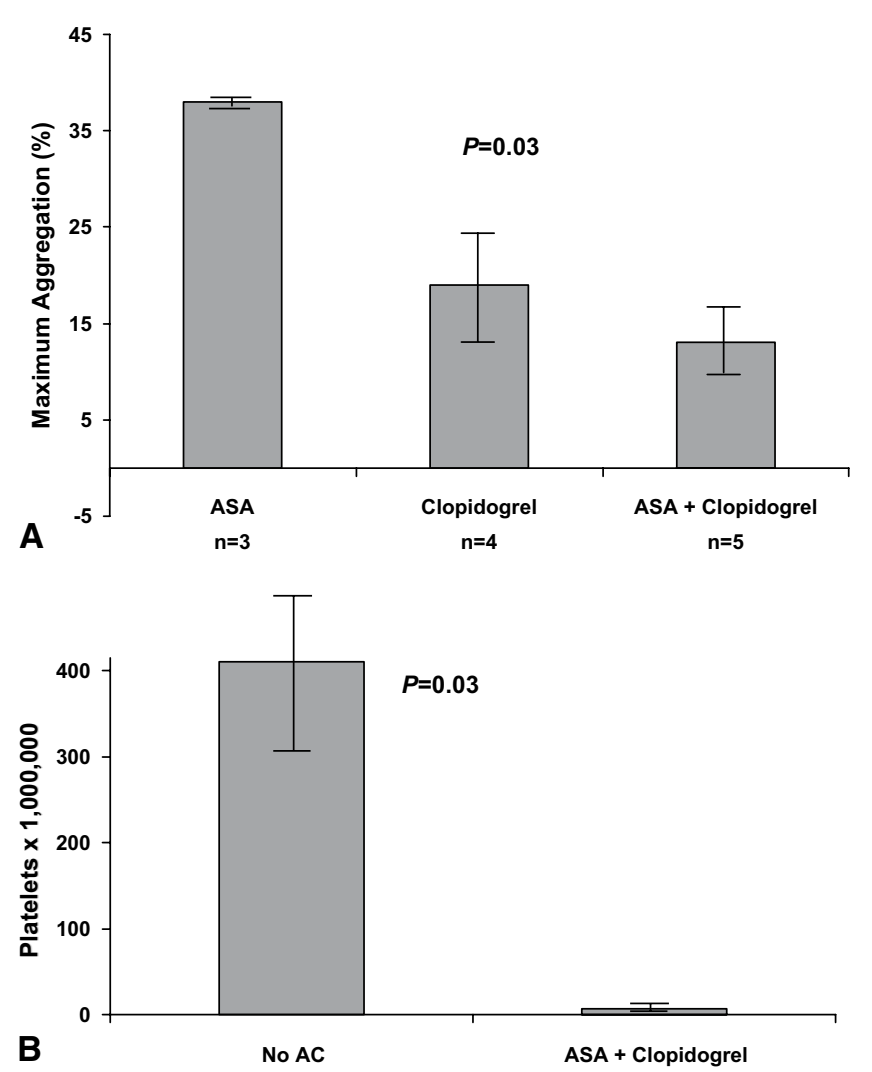

Figure 3. A, Cumulative results of aggregation in response to stimulus with $10 \mu \mathrm{mol} / \mathrm{L}$ adenosine diphosphate in platelets obtained from swine receiving antiplatelet therapy postoperatively. Data are presented as means \pm standard error of the mean. Maximum platelet aggregation decreased significantly with combination antiplatelet therapy $(P=.03)$. $B$, Mean number of platelets deposited on valves for the $\mathbf{2}$ groups: no anticoagulation and combined aspirin and clopidogrel ( $P=.03)$. $A S A$, Aspirin; $n$, number of animals; No $A C$, no anticoagulation.

in the analysis because of the inability to separate valve thrombus from vegetation. The other animal was found dead on postoperative day 66 . Necropsy was not informative as to the cause of death because thoracic, intra-abdominal, and retroperitoneal organs appeared normal; thus a lethal arrhythmia was presumed. When the valve prosthesis and aorta were examined, only $10.5 \mathrm{mg}$ of thrombus was identified on the valve and was included in the analysis. No obvious hemorrhagic or thromboembolic events were observed in the surviving 9 animals.

At 150 days, mean valve thrombus was lower for the antiplatelet group. Mean thrombus was $223 \pm 200 \mathrm{mg}$ for the no anticoagulation group and $4 \pm 4 \mathrm{mg}$ for the aspirin and clopidogrel groups $(P=.02)$, as shown in Figure 2, C. Similarly, the mean number of platelets deposited on the valve prosthesis was less for the antiplatelet group. The mean number of platelets deposited on the valves was $4.1 \times 10^{9} \pm 3.6 \times 10^{9}$ for the no anticoagulation group and $6.81 \times 10^{7} \pm 1.4 \times 10^{8}$ for the combined aspirin and clopidogrel group $(P=.03)$, as shown in Figure 3, $B$. Representative photographs of valve prostheses at 150 days are shown in Figure 4, $C$ and $D$.

\section{Discussion}

The principal finding of this study is that antiplatelet agents are effective for thromboprophylaxis of mechanical heart valves in swine. At short-term follow-up, treatment with aspirin and clopidogrel was as effective as treatment with heparin and led to the least amount of valve thrombus at 30 days. The efficacy of combined antiplatelet therapy was confirmed during long-term follow-up at 150 days.

The idea of using antiplatelet agents in place of warfarin for mechanical heart valve thromboprophylaxis is not new. Ribeiro and colleagues ${ }^{15}$ and Hartz and associates ${ }^{16}$ were among the first to report the use of combination antiplatelet agents (aspirin and dipyridamole) for thromboprophylaxis of bileaflet mechanical valves. They reported unacceptably high rates of valve thrombosis at $7.5 \%$ and $4.6 \%$, respectively. With this, enthusiasm for antiplatelet therapy diminished until more potent antiplatelet agents, such as clopidogrel, were introduced for treating cardiovascular diseases. ${ }^{17}$

Large clinical trials with combination therapy of aspirin and clopidogrel have been performed to test the hypothesis that combination antiplatelet therapy leads to more global inhibition of platelet function and improved clinical outcomes. Studies such as the Clopidogrel in Unstable Angina to Prevent Recurrent Events; Clopidogrel for High Atherothrombotic Risk and Ischemic Stabilization, Management, and Avoidance; and Clopidogrel Aspirin Stent International Cooperative trials have demonstrated the clinical benefit of combined aspirin and clopidogrel over that of aspirin alone in the prevention of vascular events, cardiovascular events, and coronary stent thrombosis, respectively. ${ }^{2,10,18}$ Mechanistically, these results might be predicted because aspirin inhibits platelet function by blocking production of thromboxane $\mathrm{A}_{2}$ through inhibition of the cyclooxygenase I enzyme. Clopidogrel, on the other hand, inhibits platelet function through an alternative pathway by inhibiting platelet ADP receptors. Our qualitative observation of decreased valve thrombus and quantitative observation of decreased platelet aggregation in animals treated with combined aspirin and clopidogrel further support this hypothesis (Figures 2 and 3).

The safety of antiplatelet therapy for mechanical heart valve thromboprophylaxis remains controversial. There is limited information on combination of antiplatelet therapy for mechanical valve thromboprophylaxis from clinical trials. Schlitt and coworkeres ${ }^{19}$ studied the combination of aspirin and clopidogrel in 11 patients undergoing aortic valve replacement with several types of mechanical prostheses. This trial was stopped prematurely after a patient with a history of pulmonary embolism experienced aortic valve 

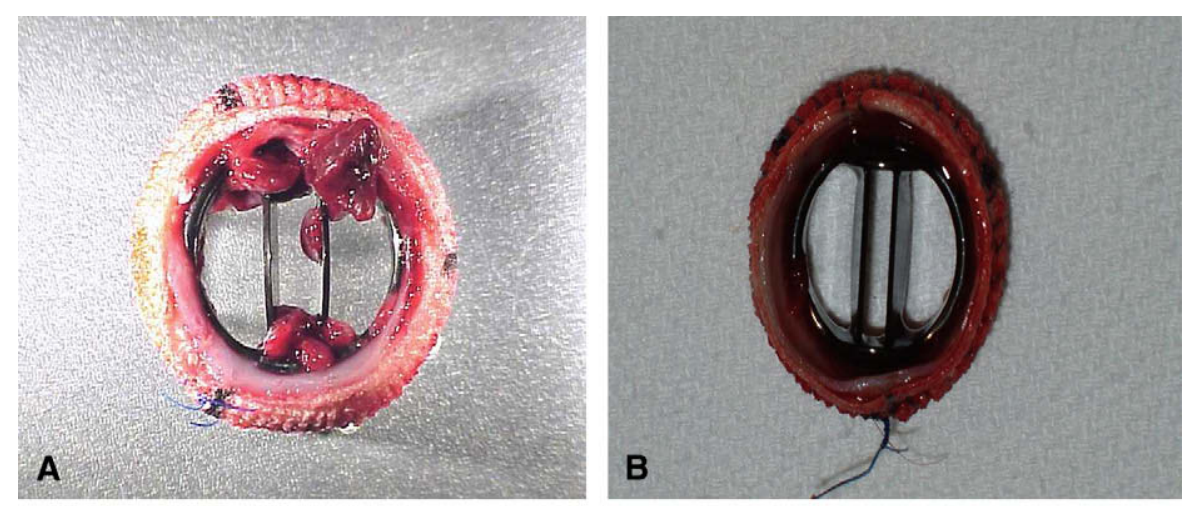

Figure 4. Postmortem photographs of explanted valves. A, Representative valve from the no anticoagulation group at $\mathbf{3 0}$ days. $B$, Representative valve from the combined aspirin and clopidogrel group at 30 days. C, Representative valve from the no anticoagulation group at 150 days. $D$, Representative valve from the combined aspirin and clopidogrel group at 150 days.
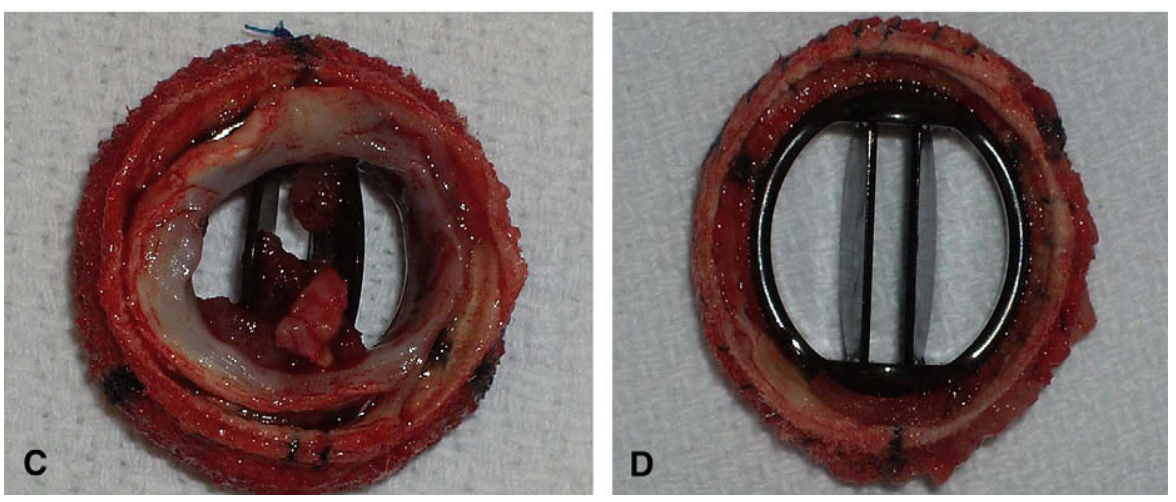

thrombosis. Inadequate platelet inhibition and an underlying prothrombotic state are possible explanations for this adverse event in light of increasing evidence of aspirin resistance and considerable interindividual variability in response to standard doses of clopidogrel. ${ }^{20}$ Aspirin resistance has been reported in up to $35 \%$ of patients, ${ }^{21}$ although its prevalence might be overestimated. ${ }^{22,23}$ Additionally, studies of platelet function in aspirin-resistant platelets demonstrate increased sensitivity to other platelet activators, such as ADP. ${ }^{24}$ Platelet function was not reported by Schlitt and coworkers, ${ }^{19}$ and it is possible that a combination of all 3 factors, aspirin resistance, ${ }^{23}$ hypersensitivity to ADP stimulation, ${ }^{24}$ and interpatient variability to clopidogrel, ${ }^{20}$ in addition to an underlying hypercoagulable state leading to the patient's prior pulmonary embolism, might have led to the valve thrombosis. Most recently, one of the authors (RGR) has more than 5 years of experience treating patients after isolated aortic valve replacement with mechanical prostheses with only combined aspirin and clopidogrel, ${ }^{25,26}$ suggesting the adverse event observed in the trial by Schlitt and coworkers ${ }^{9}$ might be quite rare. Regardless, documentation of adequate platelet inhibition as an inclusion criterion would seem essential when considering antiplatelet agents for thromboprophylaxis on mechanical valvular prostheses in human subjects and should be part of any future clinical trial. It is not clear, however, whether platelet aggregometry, as we used, or other techniques, such as thromboelastography, ${ }^{27}$ are best suited to determine platelet inhibition.

The risk of adverse bleeding events while using antiplatelet agents for thromboprophylaxis is also a potential concern. In contrast to clinical trials, ${ }^{2}$ we did not observe increased rates of hemorrhagic complications in animals receiving combined aspirin and clopidogrel therapy. We did, however, observe 3 occult hemorrhagic events in animals receiving dalteparin $(n=2)$ and aspirin $(n=1)$ in the acute study. Similarly, the 2 deaths in the chronic study were not related to antiplatelet therapy. Whether the low rates of hemorrhagic complications we observed during our studies will persist over the longer-term follow-up is unknown.

Replacing warfarin with antiplatelet agents, such as clopidogrel and aspirin, for thromboprophylaxis of mechanical heart valves has important potential clinical implications. First, clopidogrel might be safer than warfarin because of more predictable dose response and fewer drug interactions. Its action also is less affected by dietary changes, which are known to be a problem with warfarin. ${ }^{28}$ Additionally, its action is less affected by antiplatelet therapy might be more cost-effective than warfarin in that treatment does not require frequent and expensive international normalized ratio monitoring. Finally, oral antiplatelet agents might offer an advantage over intravenous or injectable heparins for short-term bridging of patients to full anticoagulation (with warfarin). 
Clinical trials can now be based on sound preclinical, large-animal data. The remaining questions relate to clinical trial design. Clinical trials could be designed to evaluate antiplatelet therapy as either initial thromboembolism prophylaxis in the immediate postoperative period or as maintenance of thromboembolism prophylaxis after 3 months of traditional anticoagulation (warfarin) therapy. Enrolling patients into a trial immediately after valve replacement surgery would attempt to answer the more robust or complicated question of prophylaxis during the period of greatest risk of thromboembolic events. ${ }^{10-13}$ In contrast, enrolling patients after 3 months of presumably more intense therapy (warfarin) might be a safer approach for evaluating dual-antiplatelet therapy because the period of greatest risk of thromboembolic events would have already passed. Perhaps the most reasonable approach would be to try to answer these 2 questions sequentially. The first trial could study combined aspirin and clopidogrel as maintenance of anticoagulation after conventional treatment (warfarin). If the results are promising and safe, a second "higher-risk" trial could enroll patients immediately after surgical intervention.

The primary limitation of the acute study was that the comparison group in the swine model was dalteparin rather than warfarin, which is used clinically. We chose dalteparin instead of warfarin because of the well-established difficulty of maintaining therapeutic anticoagulation in swine. ${ }^{12}$ The primary limitation in the chronic study was the small sample size and the lack of a full-anticoagulation control group. These were unavoidable because of the prohibitive cost of 5-month-survival animal studies and the cumbersome nature of twice-daily low-molecular-weight heparin administration, which would have been required for a heparin control group. Although greater numbers of animals would have been beneficial, the tight standard deviation observed in the mean valve thrombus weight among animals treated with aspirin and clopidogrel is encouraging and suggests that this chronic model accurately represents the biologic phenomenon we are trying to study.

\section{Conclusions}

Antiplatelet therapy with the combination of aspirin and clopidogrel is effective for short- and long-term thromboprophylaxis of mechanical heart valves. Preclinical animal data now exist and are the foundation for clinical trials evaluating antiplatelet agents as an alternative to warfarin in appropriately selected patients with bileaflet mechanical valve aortic valves.

We thank Joseph A. Rysavy, BS, for his expertise with animal care and Drs Whyte G. Owen, Krzysztof Karnicki, and Mathuvel Jayachandran for their assistance with platelet aggregation assays. We also thank St Jude Medical for providing the valved conduits for this study.

\section{References}

1. Emery RW, Krogh CC, Arom KV, Emery AM, Benyo-Albrecht K, Joyce LD, et al. The St. Jude Medical cardiac valve prosthesis: a 25year experience with single valve replacement. Ann Thorac Surg. 2005;79:776-83.

2. Bhatt DL, Fox KA, Hacke W, Berger PB, Black HR, Boden WE, et al. Clopidogrel and aspirin versus aspirin alone for the prevention of atherothrombotic events. N Engl J Med. 2006;354:1706-17.

3. Turpie AG, Eriksson BI, Lassen MR, Bauer KA. Fondaparinux, the first selective factor Xa inhibitor. Curr Opin Hematol. 2003;10:327-32.

4. O'Brien CL, Gage BF. Costs and effectiveness of ximelagatran for stroke prophylaxis in chronic atrial fibrillation. JAMA. 2005;293: 699-706.

5. Heit JA, Colwell CW, Francis CW, Ginsberg JS, Berkowitz SD, Whipple J, et al. Comparison of the oral direct thrombin inhibitor ximelagatran with enoxaparin as prophylaxis against venous thromboembolism after total knee replacement: a phase 2 dose-finding study. Arch Intern Med. 2001;161:2215-21.

6. Eriksson BI, Borris L, Dahl OE, Haas S, Huisman MV, Kakkar AK, et al. Oral, direct Factor Xa inhibition with BAY 59-7939 for the prevention of venous thromboembolism after total hip replacement. J Thromb Haemost. 2006;4:121-8.

7. Chen ZM, Jiang LX, Chen YP, Xie JX, Pan HC, Peto R, et al. Addition of clopidogrel to aspirin in 45,852 patients with acute myocardial infarction: randomised placebo-controlled trial. Lancet. 2005;366:1607-21.

8. Sabatine MS, Cannon CP, Gibson CM, Lopez-Sendon JL, Montalescot G, Theroux P, et al. Addition of clopidogrel to aspirin and fibrinolytic therapy for myocardial infarction with ST-segment elevation. N Engl J Med. 2005;352:1179-89.

9. Steinhubl SR, Berger PB, Mann JT 3rd, Fry ET, DeLago A, Wilmer C, et al. Early and sustained dual oral antiplatelet therapy following percutaneous coronary intervention: a randomized controlled trial. JAMA. 2002;288:2411-20.

10. Bertrand ME, Rupprecht HJ, Urban P, Gershlick AH. Double-blind study of the safety of clopidogrel with and without a loading dose in combination with aspirin compared with ticlopidine in combination with aspirin after coronary stenting: the clopidogrel aspirin stent international cooperative study (CLASSICS). Circulation. 2000; 102:624-9.

11. McKellar SH, Thompson JL, Schaff HV. A model of heterotopic aortic valve replacement for studying thromboembolism prophylaxis in mechanical valve prostheses. J Surg Res. 2007;141:1-6.

12. Salerno CT, Droel J, Bianco RW. Current state of in vivo preclinical heart valve evaluation. J Heart Valve Dis. 1998;7:158-62.

13. Thompson JL, Hamner CE, Potter DD, Lewin M, Sundt TM, Schaff HV. Melagatran for thromboprophylaxis after mechanical valve implantation: results in a heterotopic porcine model. $J$ Thorac Cardiovasc Surg. 2007;134:359-65.

14. Jayachandran M, Okano H, Chatrath R, Owen WG, McConnell JP, Miller VM. Sex-specific changes in platelet aggregation and secretion with sexual maturity in pigs. $J$ Appl Physiol. 2004;97:1445-52.

15. Ribeiro PA, Al Zaibag M, Idris M, Al Kasab S, Davies G, Mashat E, et al. Antiplatelet drugs and the incidence of thromboembolic complications of the St. Jude Medical aortic prosthesis in patients with rheumatic heart disease. J Thorac Cardiovasc Surg. 1986;91:92-8.

16. Hartz RS, LoCicero J 3rd, Kucich V, DeBoer A, O’Mara S, Meyers SN, et al. Comparative study of warfarin versus antiplatelet therapy in patients with a St. Jude Medical valve in the aortic position. J Thorac Cardiovasc Surg. 1986;92:684-90.

17. A randomised, blinded, trial of clopidogrel versus aspirin in patients at risk of ischaemic events (CAPRIE). CAPRIE Steering Committee. Lancet. 1996;348:1329-39.

18. Yusuf S, Zhao F, Mehta SR, Chrolavicius S, Tognoni G, Fox KK. Effects of clopidogrel in addition to aspirin in patients with acute coronary syndromes without ST-segment elevation. N Engl J Med. 2001;345: 494-502.

19. Schlitt A, von Bardeleben RS, Ehrlich A, Eimermacher A, Peetz D, Dahm M, et al. Clopidogrel and aspirin in the prevention of thromboembolic complications after mechanical aortic valve replacement (CAPTA). Thromb Res. 2003;109:131-5. 
20. Jaremo P, Starkhammar C, Lundstrom A, Lindahl TL, Richter A. Inverse relationship between the severity of gingivitis and platelet reactivity in stable angina pectoris. J Thromb Haemost. 2007;5: 422-3.

21. Andersen K, Hurlen M, Arnesen H, Seljeflot I. Aspirin non-responsiveness as measured by PFA-100 in patients with coronary artery disease. Thromb Res. 2002;108:37-42.

22. Gum PA, Kottke-Marchant K, Poggio ED, Gurm H, Welsh PA, Brooks L, et al. Profile and prevalence of aspirin resistance in patients with cardiovascular disease. Am J Cardiol. 2001;88:230-5.

23. Gum PA, Kottke-Marchant K, Welsh PA, White J, Topol EJ. A prospective, blinded determination of the natural history of aspirin resistance among stable patients with cardiovascular disease. J Am Coll Cardiol. 2003;41:961-5.
24. Macchi L, Christiaens L, Brabant S, Sorel N, Allal J, Mauco G, et al. Resistance to aspirin in vitro is associated with increased platelet sensitivity to adenosine diphosphate. Thromb Res. 2002;107:45-9.

25. Garcia-Rinaldi R. Clopidogrel-aspirin inhibit the thrombogenicity of St. Jude mechanical aortic prostheses. Ann Thorac Surg. 2006;81:787-8.

26. Garcia-Rinaldi R. Clopidogrel-ASA combination inhibits the thrombogenicity of St. Jude Medical mechanical Aortic Prostheses. Available at: http://www.ctsnet.org/doc/9565. Accessed 12-3-2007.

27. Hobson AR, Agarwala RA, Swallow RA, Dawkins KD, Curzen NP. Thrombelastography: current clinical applications and its potential role in interventional cardiology. Platelets. 2006;17:509-18.

28. Tiede DJ, Nishimura RA, Gastineau DA, Mullany CJ, Orszulak TA, Schaff HV. Modern management of prosthetic valve anticoagulation. Mayo Clin Proc. 1998;73:665-80.

Access to The Journal of Thoracic and Cardiovascular Surgery Online is reserved for print subscribers!

Full-text access to The Journal of Thoracic and Cardiovascular Surgery Online is available for all print subscribers. To activate your individual online subscription, please visit The Journal of Thoracic and Cardiovascular Surgery Online, point your browser to http://www.mosby.com/jtcvs, follow the prompts to activate your online access, and follow the instructions. To activate your account, you will need your subscriber account number, which you can find on your mailing label (note: the number of digits in your subscriber account number varies from 6 to 10 ). See the example below in which the subscriber account number has been circled:

\section{Sample mailing label}

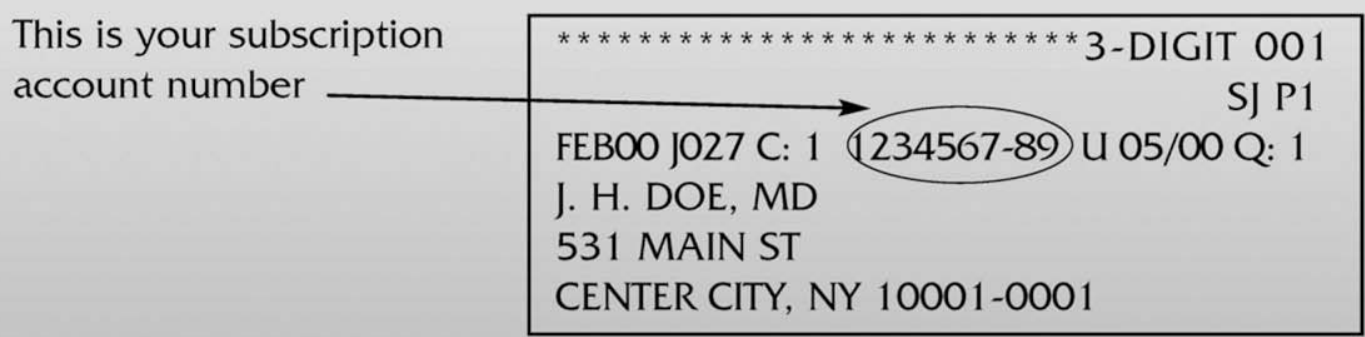

Personal subscriptions to The Journal of Thoracic and Cardiovascular Surgery Online are for individual use only and may not be transferred. Use of The Journal of Thoracic and Cardiovascular Surgery Online is subject to agreement to the terms and conditions as indicated online. 